\title{
Behavioral determinants of severe cardiac inhibition
}

\author{
JORDI CUADRAS \\ Facultat de Ciències, Universitat Autònoma, Bellaterra, Barcelona, Spain
}

\begin{abstract}
Behaviorally elicited cardiac arrests have been observed in different phyla independently of the neurogenicity or myogenicity of pacemakers. Startling stimuli such as visual detection of close and fast movement is especially associated with complete cardiac inhibition. In contrast, orienting responses are associated with bradycardia. Although studies on vertebrates are not usually concerned with reflex responses, it can be assumed that actual cardiac arrests occur in some cases in which bradycardia is reported, but that these arrests cannot be recorded because of the methodology used. Heart rate can be used to separate patterns displayed in such defensive reflex behaviors as orientation, startle, and freezing. Behavioral sequences can be categorized into various series of patterns which correspond to various sequential and multiphasic heart-rate patterns.
\end{abstract}

In a recent discussion, Obrist, Light, Langer, Grignolo, and McCubbin (1978) proposed a psychosomatic hypothesis of behavioral-cardiac interactions. This theory can be generalized to the entire kingdom, at least for groups with a definite circulatory pump. According to the theory, it can be proposed that the behavioral state of the organism interacting with sensory inputs can affect the cardiovascular system and, specifically, the heart rate. It is assumed that neuronal influences on cardiac function are of particular importance and that heart-rate adjustment associated with behavioral stressors is independent of heart-rate adjustment that is related to metabolic functions.

Some recent studies have reported the occurrence of reversible cardiac arrests (CA) in alarm patterns or startle responses in intact, unrestrained mammals, arthropods, and molluscs. Other phyla (birds and fishes) necessary to carry out an exhaustive comparative study have not received attention yet with respect to this particular field. However, the repeatability of the response in some experimental paradigms in which animals belonging to different species were faced with single (and similar) stimulation suggests a specific role of cardiac arrest in the behavioral physiology of startled animals.

Whether or not CAs are only epiphenomena without a physiological meaning can possibly not yet be determined from the results available to date, but the existence of specific cardioregulatory command fibers in some species would be a clue to the biological adaptiveness of the response. Nevertheless, the actual meaning of CA will not be well understood until the physiological and metabolic consequences are analyzed. It is implicitly assumed that the myogenicity or the neurogenicity of the heart pacemaker is not relevant here, since both kinds of oscillators are under neural regulation or control exerted by central nervous system structures in all the animals we shall be dealing with. The physiological response could have been the result of convergent evolution in separate groups and could be supported by different anatomophysiological substrates. The question proposed here concerns the regulation of a cardiovascular variable and not the mechanism of the pacemaker. CAs are instantaneous responses, and nonnervous factors such as hormones or catecholamines can be discarded.

The physiological (adaptive) CA evoked together with the startle response might evolve, sometimes, into pathological (nonadaptive), irreversible heart block, a fact which obviously adds a great deal of interest to the study of this response not only in lower animals, but also in higher mammals and human beings (Lown, Verrier, \& Rabinowitz, 1977; Obrist, Webb, Sutterer, \& Howard, 1970). This nonadaptive bias could be related to some dramatic dysfunction such as sudden infant death syndrome.

CA could be considered an extreme case of bradycardia, although CA and bradycardia are, at least in some cases, independent responses. CA can be defined as an interruption (reversible) of heart rhythm of 1 to 70 or 90 missing beats. The recovery of normal beating may show facilitation, and the postarrest rate and the basal rate do not differ. Long arrests may be interrupted by a single beat, which cannot be considered recovery of the rhythm.

Although the paper is specifically concerned with cardiac arrests, bradycardia will be considered occasionally, especially when discussing results from vertebrates. In many papers, a continuous monitoring of heart rate is not shown, and it can be assumed that actual CAs did occur but were not reported because of the use of a time-by-time methodology (beats 
per time unit), which gives only the mean value within the chosen time interval. Delays shorter than the time unit cannot be determined in these conditions. On the other hand, arrhythmia has sometimes been defined as an alteration in or absence of rhythm of the heart (Seiler, Cullen, Zimmerman, and Reite, 1979) and can include a long delay between consecutive beats (arrest).

This paper attempts to discuss what environmental inputs or behavioral changes cause CA, how these heart stoppages blend with evoked behavioral patterns, and why hearts stop under certain configurations of sensory inputs, in both invertebrates and vertebrates. Only some phyla have been studied from this point of view, but the results of different experiments are in good agreement. In the statement that some defensive patterns (or patterns displayed in defensive behavior) are general among the whole animal kingdom, similarities in concomitant visceral responses can be assumed.

\section{Cardioregulation in Crustaceans}

The literature on cardioregulation in crustaceans is, by far, the most extensive.

Wiersma and Novitski (1942) demonstrated, in dissected crayfish, the possibility of causing complete heart stoppage by direct stimulation of the cardioinhibitor fibers of Astacus with frequencies of about $40 \mathrm{~Hz}$. These cardioinhibitor fibers were under the action of an inhibitory tract leading away from the supraesophageal ganglion through the esophageal commissures and subesophageal ganglions, which showed that cardiac inhibition was centrally regulated. Florey (1960) described, in dissected crayfish and lobsters, a gradual increase of bradycardia when the frequency of stimulation of the inhibitory nerve increased, up to $35 \mathrm{~Hz}$. With higher frequencies, CAs were observed. Wilkens, Wilkens, and McMahon (1974) also reported CA in semidissected Cancer when command fibers in the circumesophageal connective were stimulated with at least $100 \mathrm{~Hz}$.

Larimer and Tindel (1966) reported CA in intact, unrestrained crayfish, which suggested that the response was not an artifact of dissected preparations. Some years later, Field and Larimer (1975a) attempted to establish some connection between the observations from intact, unrestrained animals and dissected preparations. Bradycardia or arrests were evoked by stimulation of hair sensillae on various parts of the body. Two classes of circumesophageal inhibitory interneurons have been described (Field \& Larimer, 1975b): weak inhibitors causing bradycardia and strong inhibitors eliciting CA. The strong inhibitors produced CA even when stimulated at threshold frequencies. With prolonged stimulation, the heart often escaped the inhibitory drive, as also reported by Florey (1960), Maynard (1953), and Wiersma and Novitski (1942). If the functional action of the CA is instantaneous, this escape could be a mechanism of protection against long-term inhibition, which could easily lead to an irreversible block. The weak inhibitors of Field and Larimer produced only bradycardia, even at maximal stimulation frequencies. This confirms the coexistence of two independent, but specific, inhibitory systems in crayfish. A number of strong inhibitors (Field \& Larimer, 1975b) had threshold voltages as low as $3 \mathrm{~V}$, which suggests that they were relatively large axons. This class of interneurons was effective at extremely low threshold frequencies, often less than $10 \mathrm{~Hz}$ ( $3 \mathrm{~Hz}$ in a few cases). The weak inhibitors usually required higher stimulus levels $(6-8 \mathrm{~V}, 31$ $37 \mathrm{~Hz}$ ). Nevertheless, Field and Larimer (1975b) failed to describe a behavioral pattern correlated with the CA when the cardioregulatory fibers were stimulated. Bowerman and Larimer (1974a, 1974b) tried to elicit some fixed action patterns by means of electrical stimulation of command fibers in the circumesophageal connectives of crayfish. Stimulation of fiber 150 elicited a response pattern which may be compared, in some respects to the startle response observed by Cuadras (1980) concomitant with cardiac arrests evoked by a moving target. This pattern is also similar to that evoked by stimulation of fiber CM 10 in the crayfish Procambarus (Atwood \& Wiersma, 1967), labeled the defense reflex fiber by Wiersma (1952).

If the startle response was controlled by a command interneuron, the associated cardiac and behavioral responses should be organized in higher nervous structures (supraesophageal ganglion or even optic neuropils) in a command system (according to the definition of Kupferman \& Weiss, 1978) which would innervate both motor and visceral effectors.

\section{Sensory Modalities Involved in Cardiac Inhibition}

Several authors have described spontaneous CA that is not related to any stimulation or movement (DeFur \& Mangum, 1979; Maynard, 1960; McMahon \& Wilkens, 1972; Wilkens et al., 1974). These spontaneous alterations could be related to respiratory patterns (McMahon \& Wilkens, 1972), since the majority of fibers are bivalent, synapsing on both the cardiac and the scaphognathite systems (Watson \& Wyse, 1978; Wilkens et al., 1974). In undisturbed animals of other species, CAs are only observed when leg movement or visual detection of movement (Cuadras, 1980 ) occurs, which suggests that in a completely controlled environment, heart rate decreases after manipulation (Larimer \& Tindel, 1966; Cuadras, 1980), becoming more and more regular. Actually, the fact that cardiovascular systems possess a variety of mechanisms to preserve homeostatic equilibrium, for example, through the Bohr effect, buffer activity of blood, oxygen reserve of hemocyanins and hemoglobins, variation of cardiac output through stroke 
volume, etc., suggests that absolute regularity of heart rhythms might not be essential. In some studies (Cuadras, 1980), the regularity of the heart rhythm of some crustaceans was striking after 90-120 min of rest. The basal heart rate was altered only by identifiable causes, such as, possibly, experimental stress.

Mislin (1966) described, in Astacus and Carcinus, an optocardial reflex, a complete arrest lasting up to $15 \mathrm{sec}$, caused by optical stimuli, and Larimer and Tindel (1966) and Cuadras (Note 1) observed that, in crayfish and hermit crabs, respectively, darkening the environment resulted in bradycardia but not in true CA. This response can be interpreted as orientation. Larimer and Tindel (1966) observed CA when crayfish perceived the presence of food (chemical sensing or contact). These authors did not describe heartrate fluctuations in animal-animal interactions, but some evidence has been found that the visual detection of movement of a conspecific animal produces CA (Cuadras, 1979; Florey \& Kriebel, 1974). Stimulation to produce CA (touching eyes, antennae, mouthparts, legs, or carapace) was ineffective if the bottom of the tank was covered with a layer of fine gravel that would allow the crabs to burrow into it (Florey \& Kriebel, 1974), but visual detection of movement was effective in stopping the heart in sandy-bottom tanks (Cuadras, 1979, 1980). In agreement with results reported by other authors, Uglow (1973) described CA caused in portunid crabs by visual stimuli, as well as by vibrational stimuli. Cuadras (1980) failed to cause CA with vibrational stimuli.

Often observations do not take into account the behavioral response to the stimulation presented. CA and some forms of bradycardia could be concomitant with some behavioral patterns, and thus the type of stimulation may be more or less unimportant. A moving target elicited CA in Cancer (Wilkens et al., 1974) and in Dardanus (Cuadras, 1980), in the latter case associated with a startle response. When the crabs were approached by observers, bradycardia (Depledge, 1978) or CA (Florey \& Kriebel, 1974), as well as startle (Cuadras, 1979), occurred. When behavior was also recorded, CA appeared to be a physiological component of the startle response (Cuadras, 1980, Note 1), regardless of the ensuing pattern of behavior and specific cardiac activity that usually followed.

\section{Molluses}

CA has been studied exhaustively only in crustaceans. Insects and vermes perhaps provide inadequate material, but molluscs have received some attention. Results from a few isolated studies suggest that the patterns evidenced in molluscs possibly resemble those in crustaceans.

Evidence for two regulatory modalities (excitation and inhibition) in the hearts of molluscs has also been provided (see review by Koester et al., 1979, and Wells, 1980).
MacKay and Gelperin (1972) found that touching the head of Limax produced cardiodeceleration, whereas touching the caudal area evoked acceleration. CA induced by sudden stimuli have been reported in Octopus (Wells, 1979). No exhaustive analysis of behavioral responses has been done on cephalopods, but some connection between startle and CA has been suggested. As was the case for crustaceans, when a Perspex rectangle was detected, CA resulted, and habituated if repeated. CAs have also been reported during sexual displays of Octopus (Wells, 1979) when a female moved toward the recording subject. This result must be compared with Cuadras's (1979) findings with respect to social interactions: The release for CA could be the perceived movement, independently of the nature of the evoked behavior (sexual or aggressive). Furthermore, visual detection of motion seems to be, again, the input most relevant in causing CA. Wells (1979) has also observed CA at each ejaculation. This particular cardiac inhibition may depend on several factors, some of which are only physiological. In this case, attributing inhibition to an ethological cause would be imprudent; the situation seems to be more comparable to heart stoppage caused by movements of the alimentary canal observed in several insects (Jones, 1974).

\section{The Psychobiology of CA in Invertebrates}

CA is a response supported by a specific anatomophysiological background, a neural pathway with low thresholds and possibly large axons. This fact suggests that CA systems have evolved to produce fast responses to certain novel stimuli, even when those stimuli are detected at low intensities. The escape of the heart under sustained stimulation would ensure that the response is kept under control. CAs appear to be organized responses and cannot be interpreted as epiphenomena that have no physiological meaning. It can be assumed that, whereas bradycardia is usually elicited together with orienting responses, CA is concomitant with startle patterns. Yet, the nature of startling stimuli is not well understood. The most conspicuous startle stimulus is visual stimulation, specifically, visual detection of sudden movement. In the laboratory, a target moving toward the animal or passing nearby can act as a releaser or signal stimulus for both the startle response and CA, and the specificity of the cardiac response, in addition to its anatomophysiological support, indicates a specific function for cardiac inhibition in startling, as well as for bradycardia in orientation. Moreover, differences in cardiac rhythms can be used to identify behavioral patterns (see Figure 1).

CAs have also been observed when movement, locomotion, and even organized complex behaviors are displayed (Cuadras, 1979, 1980; Maynard, 1960; Needham, 1954). Since this cardiac activity could have behavioral implications, some discussion ap- 

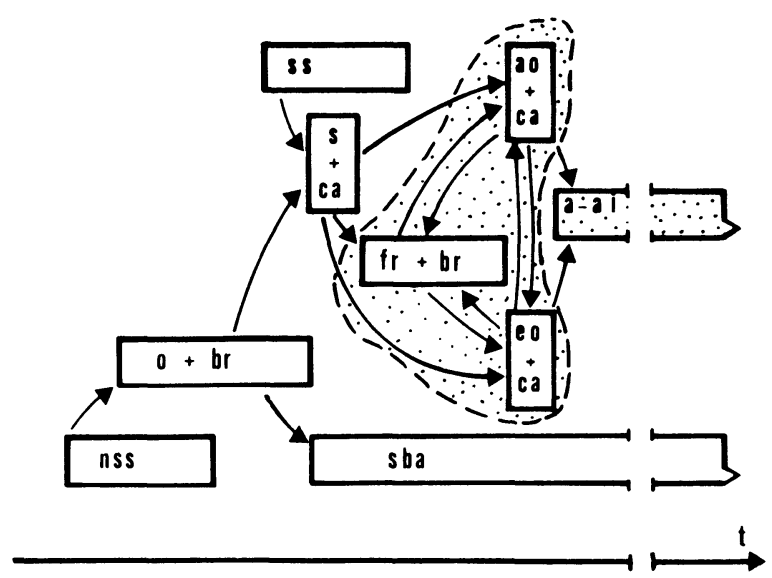

Figure 1. Model for temporal organization of patterns involved in defensive behavior. The relative durations of visceromotor patterns are represented along the abscissa. See text for more details.

pears to be necessary. Larimer and Tindel (1966) suggested that rapid movement of the abdomen might fill the pericardium and thus lead to a reflex bradycardia. Cuadras (1980) observed that imposed movements of the chelipeds of hermit crabs (Cuadras, 1980) prolonged cardiac inhibition, even though the hydrodynamic effect of the movement can be detected only at the onset of the movement. Furthermore, the above authors' interpretations seem inadequate to an understanding of the long arrests observed in complex integrated patterns that are not associated with sudden movements. On the other hand, CA concomitant with movement often seems to precede the onset of muscular activity and therefore cannot be considered a consequence of the movement. Nevertheless, such striking cardiac inhibition will not be well understood until an exhaustive investigation of the connection between proprioception and inhibitory interneurons is carried out.

In general, only motor components of defensive reflexes have been analyzed (defensive withdrawal reflex of Aplysia, escape behavior of crayfishes and lobsters, etc.). Insight into visceral activity and into its metabolic effects may provide greater knowledge of the organization of behavior. In the case of the startle reflex in hermit crabs, it has been shown that the thoracicoabdominal response is the first to habituate, followed by cardiac arrest and finally by an antennular response of very slow, if any, habituation (Cuadras, 1980). These results suggest not only the nervous organization of the habituation of several elements of the behavioral pattern, but also the following: First, short-term recovery (dishabituation) is a property of lower animals and cannot be taken as a characteristic of the response (obviously, in higher animals, behavioral changes are more persistent). Second, what is the significance of the differential habituation? When startle and CA have habituated, antennular response is still present. Antennular response could be an element of orientation. Obviously, a startling stimulus that loses its potential for danger (familiarization) can still require orientation. When no component has as yet habituated, startle and orientation will be confounded. It can be concluded that (1) CA is not a component of orientation but of startle, and (2) CA is not a consequence of muscular response. When motor startle is habituated and no longer recorded, a CA can still be produced, which may suggest that some physiological changes are still necessary.

\section{Experimental Data From Vertebrates}

A large body of data dealing with heart-rate alterations caused by psychological stress in higher vertebrates is available (see review by Cohen \& Obrist, 1975), and even a cause-effect relationship between psychological factors and sudden cardiac death in man and animals has been suggested by some authors (Henry, 1973; Lown et al., 1977; Obrist et al., 1970) who seek new insights into cardiopathy by examining the role of the higher nervous system in chronotropic modifications of heart activity. The experimental paradigms used by most of the authors are concerned with chronic stress, or its effects, and include conditioning, avoidance tasks, anticipation (Cohen, 1974b; Cohen \& Obrist, 1975; Gantt, 1960), maternal separation (Seiler et al., 1979), and social status (Candland, Bryan, Nazar, Kopf, \& Sendor, 1970). The physiological meaning of heart arrests concomitant with startle could be absolutely different from that of cardiovascular adaptations over a period of hours or days. On the other hand, both CA and longterm stress can lead to cardiac dysfunctions caused by a lack of circulation in the former case and by myocardial degeneration (Corley, Mauck, \& Shiel, 1975; Corley, Mauck, Shiel, Barber, Clark, \& Blocher, 1979; Corley, Shiel, Mauck, Clark, \& Barber, 1977; Mascitelli-Coriandolli, 1958; Raab, Chaplin, \& Bajusz, 1964) involving hormonal factors in the latter. Only nervous regulation is thought to be responsible for CA.

The autonomic nervous system and central areas related to cardiac regulation (acceleration and deceleration) are well known in higher vertebrates, and the neural pathways involved in specific cardiovascular responses to behavior are beginning to be understood (Cohen, 1974a, 1974b). Nevertheless, a description of the interaction of sympathetic and vagal activity to produce a slowed heart rate has proved difficult to achieve in spite of the volume of work in this field (Cohen, 1974; Obrist, Howard, Lawler, Sutterer, Smithson, \& Martin, 1972; Obrist, Lawler, Howard, Smithson, Martin, \& Manning, 1974; Obrist \& Webb, 1967; Obrist et al., 1970; Obrist, Wood, \& Perez-Reyes, 
1965; Sampson, Schneiderman, Wallach, Gavin, \& Francis, 1977, etc.). These works enable a general conclusion: Sympathetic and parasympathetic extrinsic innervation of the heart act synergically, but vagal activity (which can mask sympathetic activity) is of a primary importance in chronotropism of the heart, although sympathetic activity is important in other cardiovascular parameters.

Studies by Reite (1977), Reite and Short (1978), Reite, Short, Kaufman, Stynes, and Pauley (1978), and Seiler et al. (1979) have demonstrated arrhythmias and CA apparently not caused by direct stimulation of young monkeys stressed by maternal separation. Yoked monkeys not allowed to predict or control electroshock exhibited severe bradycardia that led to ventricular arrests (Corley, Shiel, Path, Mauck, \& Greenhoot, 1973; Corley et al., 1975; Corley et al., 1979; Corley et al., 1977.)

In a pioneer study by Richter (1957) inspired by earlier work by Cannon (1942), sudden deaths of wild rats held in the hand or put into water were reported. Deaths occurred following irreversible CA (heart in diastole), sometimes after a period of severe bradycardia. As for the case of voodoo death (Cannon, 1942), hopelessness seems to be a crucial factor in cardiac arrest. The rats of Richter (1957) were in a situation for which they had no defense, and, in fact, their familiarization with the experimental methodology resulted in reduced numbers of deaths. In several attempts to pursue the work by Richter (1957), Binik, Deikel, Theriault, Shustack, and Balthazard (1979), and Rosellini, Binik, and Seligman (1976) suggested a close relationship between the diving reflex and bradycardia developing into asystole. Anoxia could mediate dramatic heart-rate alterations and thus lead to death. The behavioral cause of CA is thus unclear.

Bohus (1974) reported a decrease in the heart rate of rats during passive avoidance to electric shock: the higher the shock intensity, the more pronounced the bradycardia. Rosellini et al. (1976) suggested that housing and handling might cause sudden deaths in laboratory rats, and a study by Hofer (1970) demonstrated that wild rodents placed suddenly into a stressful environment exhibited cardiac arrhythmias and bradycardia.

As soon as a moving target was presented to deer mice, Peromyscus maniculatus bairdii (Rosenmann \& Morrison, 1974), alarm responses and pronounced bradycardia that closely correlated with decreases in oxygen consumption developed.

Startled young rats exhibited heart-rate deceleration (Hofer, 1974). Jacobsen (1979) noted that young fawns (Odocoileus) responded to alarm stimuli with either bradycardia, during passive reactions, or by lying prone. Actual CAs were not described, but Espmark and Langvatn (1979) reported CAs of up to 10 missed beats, elicited by startling stimuli such as an approaching man or stones being thrown and landing 4-5 $\mathrm{m}$ from the animals, in young red deer calves (Cervus). In animal-animal interactions, heart blocks of up to $3 \mathrm{sec}$ were observed. Thus, similar stimulations produce similar responses in some crustaceans, molluscs, and mammals. Habituation of the cardiac responses of calves was also suggested, although an analysis of the habituation of motor and cardiac responses was not provided. As the above authors have noted, both the behavioral responses to sudden stimuli, ranging from startling-freezing in young animals to flight in adults, and the autonomic responses to those stimuli, including changes in heart rate (Hofer, 1974), should be considered. The startle pattern appears during the entire life of animals, although what constitutes startling stimuli could be modified by the experience of individuals (learning) or species (communication). If CA always accompanied startle, independently of the following response (freezing, flight, withdrawal, or attack), evidence on behalf of the specificity of its function would have to be accepted.

Adams, Baccelli, Mancia, and Zanchetti (1971) observed bradycardia when cats faced a stranger and were preparing to fight, but tachycardia when the stranger threatened. Rabbits exhibiting the "freeze" response also evidenced bradycardia (Schneiderman, Francis, Sampson, \& Schwaber, 1974).

Carrol and Rhys-Davies (1979) studied exercise paradigms in man. They reported a slight decrease in heart rate at the very beginning of exercise, a decrease observable thanks to a second-by-second analysis. The heart-rate decrease could be related to an orienting response to the signal to start exercising. Although no relationship exists between this signal and a potentially noxious startling stimulus, the heart response should be discussed in the context of a sudden muscular activity that, in some instances, might be compared to startle.

Contrary to the general results, the heart of Parus major accelerated when a startle reflex was elicited by an alarm call (auditory stimulus) (Ryden, 1980), a fact which again indicates some specificity for stimulation. In fact, generalizations may be made only with respect to one sensory modality, the visual perception of close and sudden movement.

\section{A Model for the Relationship Between Defensive Patterns and Cardiac Inhibition}

In strictly controlled experimental studies with sensory-isolated, intact, unrestrained, and nonstressed animals, under simulated-natural conditions, CAs appear only after stimulation or movement. Visually perceived movement is of primary importance in evoking CA, independently of whether the context is social, aggressive, or sexual.

Heart deceleration has been related to orientation 
(Graham \& Clifton, 1966; Lacey, Kagen, Lacey, \& Moss, 1963; Lacey \& Lacey, 1958) or associated with enhanced sensitivity to stimulation and attention in decision-making processes, and the startle reflex could facilitate behavioral intensity (Andrew, 1974) by acting on nervous responsiveness (arousal). Orientation is elicited by nonspecific, nonnoxious, nonsignal stimuli and is defined as a variable movement of parts of the body (receptor organs, head) to cope with a bigger and better influx of information. Orientation reflex may involve only a few, short movements, or it may be a generalized focusing of sensory organs. This reflex is then replaced by a subsequent behavior pattern evoked by the new sensory inputs configuration. Subsequent patterns include alarm, hesitation, fight, flight, feeding, cleaning, etc. In addition to a number of well-established physiological changes, orientation seems to be concomitant with deceleration of the heart rate. In startling, the very first response may be one of orientation, which is masked by both violent motor and visceral responses, including CA. Upon habituation of the startle, only an orientational component persists, and it has a much slower habituation.

In some cases (Hoffman \& Fitzgerald, 1978), an opposite heart-rate orientation response to noxious stimuli (electric shock, ammonia fumes) has been observed. In this case, in addition to the question of the specificity of orientation to various stimuli, the various neural pathways reporting the different sensory information should be taken into account. On the other hand, the heart response may here constitute some alarm pattern.

Orientation and startle have of ten been confounded, but, as CA is a component of startle but not of orientation, heart rate can be used to separate these responses. Some precautions have to be taken, however. Results from different authors have been compared under the assumption that experimental stimulations were similar. But, in fact, cardiovascular responses of different natures could have been lumped together. For instance, the same mechanical stimulus applied to different areas of a mollusc can elicit avoidance or escape (McKay \& Gelperin, 1972), and these responses could be associated with different cardiac activities. The stimulation may be nonspecific per se but, depending on the configuration of inputs, modulated by the behavioral state (responsiveness) of the animal.

A search for all possible stimulatory modalities that may evoke CA would be a valuable contribution. Unfortunately, visual detection of movement is the only modality that allows, at this time, an analysis of the heart rhythm.

CAs have been observed at the onset of movements, but many results suggest bradycardia when animals flee or engage in a prolonged attack, al- though this bradycardia is then interrupted by CA if the animals are stimulated.

Behavioral sequences of patterns in defensive behavior can be organized into various series of units, such as startling, freezing, hesitation, flight, fight, orientation, etc.; various sequential but multiphasic heart rates may be recorded, and each heart-rate pattern could serve to identify these patterns as shown in Figure 1.

A nonstartling stimulus (nss) elicits only an orientation response with a bradycardia component $(\mathrm{o}+$ br). Orientation may be followed by exploration or by any specific behavioral arousal (sba) pattern, such as feeding, etc. The amount of new information obtained via the orientation reflex, or the new input configuration, may evoke a startle accompanied by cardiac arrest $(\mathrm{s}+\mathrm{ca})$. Obviously, startle and arrest may be directly elicited by a startling stimulation (ss). Orientation may continue after stimulation, but startle is a fast response with a short delay and may conclude before the stimulus does. Startling may lead to freezing (fr) with a bradycardia component (br) or to brief movements of escape or attack, usually occurring together with cardiac arrest $(\mathrm{ao}+\mathrm{ca}, \mathrm{eo}+\mathrm{ca})$. A similar response is evoked by visual perception of such brief movements by another animal. Freezing begins while the stimulus is still acting and may last longer than the stimulation. After stimulus disappearance, freezing can stop. Each temporal organization of freezing, attack, or escape onset constitutes a hesitation pattern (stippled area in Figure 1), which may develop into close complex animal-animal interactions (a-ai) involving arrhythmias and arrests, in addition to tachycardia. If not resolved, hesitation and some kinds of animal-animal interactions may lead to chronic stress. Cardiopathy may then ensue.

The basal heart rate is not considered. In studying crustaceans, some authors began recording after only a few hours, whereas others felt that a period of 24-48 $\mathrm{h}$ after electrode implantation was necessary for recovery of the actual basal heart rate. Experimental stress may have had an effect on basal heart rate and thus on the occurrence of CA, and could be very important in long-term paradigms. The model does not deal with long-term paradigms in which long-term stress could mask a direct stimulus-response relationship. Responsiveness to startling stimuli as a function of initial heart rate should also be considered, since it is known that initial values can interfere with the poststimulus heart rate (Richards, 1980). The model does not consider learning factors. Familiarization probably alters responsiveness to specific stimulation. Habituation and some cases of conditioning to enhance responsiveness could be viewed as modifications of the properties of stimuli by means of converting a nonstartling stimulus into a startling one, or vice versa. 


\section{On the Biological Meaning of Cardiac Arrests}

Although a close relationship between heart rates and some fixed action patterns can be discerned, that does not define the reasons for variations in heart rates. Some authors (MacMahon \& Wilkens, 1972) have suggested that CA could represent concealment from predators or prey that might be able to detect the regular electrical or pressure gradients associated with cardiac or branchial pumping. But CAs are also observed in terrestrial animals living in a nonaquatic medium (air) inadequate for conducting electrical or pressure potentials. It is true that complete immobility (including cessation of respiratory movements) could be a good defensive strategy, but pauses in cardiac rhythms appear to be rather useless as an aid to concealment.

Although cardiac responses can be divorced from somatic processes (Miller \& Dicara, 1967), a decrease in somatic activity is often accompanied by a decrease in heart rate (Obrist, Webb, \& Sutterer, 1969), a fact that suggests some coupling between both motor and visceral systems. Obviously, when the heart is inhibited by sensory inputs that elicit a behavioral pattern, some degree of specificity or collateral coupling is needed to organize and coordinate the response, but CAs are not a consequence of the muscular activity, although they are concomitant with it.

The utility of CA should be studied in terms of its physiological consequences: Although cardiovascular acceleration can easily be understood in terms of preparing for great oxygen consumption and of the necessity for rapid blood flow to eliminate waste products, no easy interpretation of deceleration can be proposed. McMahon and Wilkens (1972) reported a decrease in blood oxygen levels of crustaceans $\left(\mathrm{PO}_{2}=50-60 \mathrm{~mm} \mathrm{Hg}\right.$ down to $27 \mathrm{~mm} \mathrm{Hg}$ in postbranchial blood, with external oxygen levels of $80-120 \mathrm{~mm} \mathrm{Hg}$, and down to $9.5 \mathrm{~mm} \mathrm{Hg}$ in prebranchial blood). This decrease can supersede the Bohr effect and may act as an agent in subsequent metabolic and physiologic action in central nervous system areas sensitive to hypoxia. In animals with only one systemic heart, when blood flow stops, tissue respiration follows (with the possibility of an oxygen debt) and hypercarbia and consequent acidosis can ensue, due to the failure of branchial elimination of $\mathrm{CO}_{2}$. In fact, handling has been reported to cause acidosis (Butler, Taylor, \& McMahon, 1978). But handling can cause chronotropic modifications in the heart, such as arrhythmias and arrests, which could in turn cause the reported acidosis. More direct evidence of bradycardia-induced decreases in blood $\mathrm{pH}$ has been obtained from experiments dealing with bradycardia associated with diving in aerial animals (Lillo, 1978).

If blood flow stops, blood pressure falls, which changes the hydrodynamics of tissue irrigation, as well as the equilibrium of metabolic transfer and transportation of information molecules. Unfortunately, we do not know the importance of these alterations (or even if there are any) in the physiology of the nervous system specifically concerned with startle, orientation, and other patterns in reflex responses to novel stimuli.

\section{REFERENCE NOTE}

1. Cuadras, J. Orienting and alarm in a crustacean: Sensory appendages and heart coordination. Manuscript in preparation, 1981.

\section{REFERENCES}

Adams, D. B., Baccelli, G., Mancia, G., \& Zanchetti, A. Relation of cardiovascular changes in fighting to emotion and exercise. Journal of Physiology, 1971, 212, 321-335.

Andrew, R. J. Arousal and the causation of behaviour. Behaviour, 1974, 51, 135-165.

Atwood, H. L., \& Wiersma, C. A. G. Command interneurons in the crayfish central nervous sytem. Journal of Experimental Biology, 1967, 46, 249-261.

Binik, Y. M., Deikel, S. M., Theriault, G., Shustack, B., \& Balthazard, C. Sudden swimming deaths: Cardiac function, experimental anoxia and learned helplessness. Psychophysiology, 1979, 16, 381-391.

Binik, Y. M., Theriault, G., \& Shustack, B. Sudden death in the laboratory rat: Cardiac function, sensory and experimental factors in swimming deaths. Psychosomatic Medicine, 1977, 39, 82-92.

Bohus, B. Telemetered heart rate responses of rat during free and learned behaviour. Biotelemetry, 1974, 1, 193-201.

Bowerman, R. F., \& Larimer, J. L. Command fibres in the circumoesophageal connectives of crayfish, I: Tonic fibres. Journal of Experimental Biology, 1974, 60, 95-117. (a)

Bowerman, R. F., \& Larimer, J. L. Command fibres in the circumoesophageal connectives of crayfish, II: Phasic fibres. Journal of Experimental Biology, 1974, 60, 119-134. (b)

Butler, P. J., TAylor, E. W., \& McMahon, B. R. Respiratory anc circulatory changes in the lobster Homarus vulgaris during long term exposure to moderate hypoxia. Journal of Experimental Biology, 1978, 73, 131-146.

Candland, D. K., Bryan, D. C., Nazar, B. L., Kopf, K. J., \& SENDOR, M. Squirrel monkey heart rate during formation of status orders. Journal of Comparative and Physiological Psychology, 1970, 70, 417-423.

Cannon, W. D. "Voodoo" death. American Anthropologist, $1942,44,169-181$.

Carroll, D., \& Rhys-Davies, L. Heart rate changes with exercise and voluntary heart rate acceleration. Biological Psychology, $1979,8,241-252$.

Conen, D. H. Analysis of the final common path for heart rate conditioning. In P. A. Obrist, A. H. Black, J. Brener, \& L. V. Dicara (Eds.), Cardiovascular psychophysiology: Current issues on response mechanisms, biofeedback and methodology. Chicago: Aldine, 1974. (a)

Conen, D. H. The neural pathways and informational flow mediating a conditioned autonomic response. I L. V. Dicara (Ed.), Limbic and autonomic nervous system research. New York: Plenum Press, 1974. (b)

Conen, D. H., \& ObRist, P. A. Interactions between behavior and the cardiovascular system. Circulation Research, 1975, 37, 693-706. 
Corley, K. C., Mauck, H. P., \& Shiel, F. O'M. Cardiac responses associated with "yoked-chair" shock avoidance in squirrel monkeys. Psychophysiology, 1975, 12, 439-444.

Corley, K. C., Mauck, H. P., Shiel, F. O'M., Barber, J. H., Clark, L. S., \& Blocher, C. R. Myocardial dysfunction and pathology associated with environmental stress in squirrel monkey: Effect of vagotomy and propanolol. Psychophysiology, $1979,16,554-560$.

Corley, K. C., Shiel, F. O'M., Mauck, H. P., Clark, L. S., \& BARBer, J. H. Myocardial degeneration and cardiac arrest in squirrel monkey: Physiological and psychological correlates. Psychophysiology, 1977, 14, 322-328.

Corley, K. C., Shiel, F. O'M., Path, M. R. C., Mauck, H. P., \& Greenhoot, J. Electrocardiographic and cardiac morphological changes associated with environmental stress in squirrel monkey. Psychosomatic Medicine, 1973, 35, 361-364.

Cuadras, J. Heart rate and agonistic behaviour in unrestrained crabs. Marine Behavior \& Physiology, 1979, 6, 189-196.

CuAdras, J. Cardiac responses to visual detection of movement, mechanostimulation and cheliped imposed movement in hermit crabs. Comparative Biochemistry and Physiology, 1980, 66, 113-117.

Defur, P. L., \& Mangum, C. P. The effects of environmental variables on the heart rates of invertebrates. Comparative Biochemistry and Physiology, 1979, 62, 283-294.

DEPLEDGE, M. H. Cardiac activity of the shore crab Carcinus maenas. Comparative Biochemistry and Physiology, 1978, 60, 65-67.

Espmark, Y., \& Langvatn, R. Cardiac responses in alarmed red deer calves. Behavioral Processes, 1979, 4, 179-186.

Field, L. H., \& Larimer, J. L. The cardioregulatory system of crayfish: Neuroanatomy and physiology. Journal of Experimental Biology, 1975, 62, 519-530. (a)

Field, L. H., \& Larimer, J. L. The cardioregulatory system of crayfish: The role of circumoesophageal interneurones. Journal of Experimental Biology, 1975, 62, 531-543. (b)

FLOREY, E. Studies on the nervous regulation of the heart beat in Decapod Crustacea. Journal of General Physiology, 1960, 43, 1061-1081.

Florey, E., \& Kriebel, M. E. The effects of temperature, anoxia and sensory stimulation on the heart rate of unrestrained crabs. Comparative Biochemistry and Physiology, 1974, 48, 285-300.

GaNTT, W. H. Cardiovascular component of the conditional reflex to pain, food and other stimuli. Physiological Review, 1960, 40, 266-291.

Graham, F. K., \& Clifton, R. K. Heart rate change as a component of the orienting response. Psychological Bulletin, 1966, 65, 305-320.

HENRY, J. P. La psychophysiologie des maladies du coeur. Recherche, 1973, 4, 771-779.

Hofe R, M. A. Cardiac and respiratory function during sudden prolonged immobility in wild rodents. Psychosomatic Medicine, $1970,32,633-642$.

HoFe R, M. A. The role of early experience in the development of autonomic regulation. In L. V. Dicara (Ed.), Limbic and autonomic nervous system research. New York: Plenum Press, 1974.

Hoffman, J. W., \& Fitzgerald, R. D. Classically conditioned heart rate and blood pressure in rats based on either electric shock or ammonia fumes reinforcement. Physiology \& Behavior, 1978, 21, 735-741.

JACOBSEN, N. K. Changes in heart rate with growth and activity of white-tailed deer fawns, Odocoileus virginianus. Comparative Biochemistry and Physiology, 1979, 62, 885-888.

JoNES, J. C. Factors affecting heart rate in insects. In M. Rockstein (Ed.), The physiology of insects. New York: Academic Press, 1974.

Koester, J., Dieringer, N., \& Mandelbaum, D. E. Cellular neuronal control of molluscan heart. American Zoologist, 1979, 19, 103-116.
Kupfermann, I., \& Weiss, K. R. The command neuron concept. Behavior and Brain Science, 1978, 1, 3-39.

Lacey, J. I., Kagen, J., Lacey, B. C., \& Moss, H. A. Situational determinants and behavioral correlated autonomic response patterns. In P. J. Knapp (Ed.), Expression of the emotions in man. New York: International Universities Press, 1963.

LACEY, J. I., \& LACEY, B. C. The relationship of resting autonomic activity to motor impulsivity. In The brain and human behaviour (Proceedings of the Association for Research on Nervous and Mental Disorders). Baltimore, Md: Williams \& Wilkins, 1958.

LARimer, J. L., \& Tindel, J. R. Sensory modifications of heart rate in crayfish. Animal Behaviour, 1966, 14, 239-245.

LiLlo, R. S. The effect of arterial blood $\mathrm{pO}_{2}$ and $\mathrm{pH}$ on diving bradycardia in the bullfrog Rana catesbeiana. Physiological Zoology, 1978, 51, 340-346.

Lown, B., Verrier, R. L., Rabinowitz, S. H. Neural and psychologic mechanisms and the problem of sudden cardiac death. American Journal of Cardiology, 1977, 39, 890-902.

Mackay, A. R., \& Gelperin, A. Pharmacology and reflex responsiveness of the heart in the giant garden slug Limax maximus. Comparative Biochemistry and Physiology, 1972, 43, 877-896.

McMahon, B. R., \& Wilkens, J. L. Simultaneous apnoea and bradycardia in the lobster Homarus americanus. Canadian Journal of Zoology, 1972, 50, 165-170.

Mascitelli-Coriandoli, E. Cardiac damage caused by experimental stress. Nature (London), 1958, 181, 1215-1216.

MAYNARD, D. M. Activity in a crustacean ganglion. I: Cardioinhibition and acceleration in Panulirus argus. Biological Bulletin of Marine Biology Laboratory, Woods Hole, 1953, 104, 156-170.

MaYnARD, D. M. Circulation and heart function. In T. H. Waterman (Ed.), Physiology of crustacea. New York: Academic Press, 1960.

Mille R, N. E., \& Dicara, L. V. Instrumental learning of heart rate changes in curarized rats: Shaping and specificity to discriminative stimulus. Journal of Comparative and Physiological Psychology, 1967, 63, 12-19.

Mislin, H. Experimenteller Nachweis der Beeinflussung des Elektrokardiogramms dekapoder Krebse (Astacus fluviatilis, Astacus leptodactylus, Carcinus maenas) durch ootishe Reize (Optocardialer hemmreflex). Review of Swiss Zoology, 1966, 73, 301-312.

Needham, A. E. Physiology of the heart of Asellus aquaticus. Nature (London), 1954, 173, 272.

Obrist, P. A., Howard, J. L., Lawler, J. E. Sutterer, J. R., Smithson, K. W., \& Martin, P. L. Alterations in cardiac contractility during classical aversive conditioning in dogs: Methodological and theoretical implications. Psychophysiology, 1972, 9, 246-261.

Obrist, P. A., Lawler, J. E., Howard, J. L., Smithson, K. W., Martin, P. L., \& Manning, J. Sympathetic influences on cardiac rate and contractility during acute stress in humans. Psychophysiology, 1974, 11, 405-427.

Obrist, P. A., Light, K. C., Langer, A. W., Grignolo, A., \& McCubbin, J. A. Behavioural-cardiac interactions: The psychosomatic hypothesis. Journal of Psychosomatic Research, 1978, 22, 301-325.

Obrist, P. A., \& Weвb, R. A. Heart rate during conditioning in dogs: Relationship to somatic-motor activity. Psychophysiology, 1967, 4, 7-34.

Obrist, P. A., Webb, R. A., \& Sutterer, J. R. Heart rate and somatic changes during aversive conditioning and a simple reaction time task. Psychophysiology, 1969, 5, 696-723.

Obrist, P. A., Webb, R. A., Sutterer, J. R., \& Howard, J. L. The cardiac-somatic relationship: Some reformulations. Psychophysiology, 1970, 6, 569-587.

Obrist, P. A., Wood, D. M., \& Perez-Reyes, M. Heart rate during conditioning in humans: Effects of UCS intensity, vagal blockade, and adrenergic block of vasomotor activity. Journal of Experimental Psychology, 1965, 70, 32-42. 
RaAb, W., Chaplin, J. P., \& B ajusz, E. Myocardial necroses produced in domesticated rats and wild rats by sensory and emotional stresses. Proceedings of the Society for Experimental Biology and Medicine, 1964, 116, 665-669.

REITE, M. Maternal separation in monkey infant: A model of depression. In I. Hanin \& E. Usdin (Eds.), Animal models in psychiatry and neurology. Oxford, Pergamon, 1977.

REITE, M., \& Short, R. A. Nocturnal sleep in separated monkey infants. Archives of General Psychiatry, 1978, 35, 1247-1253.

Reite, M., Short, R., Kaufman, I. C., Stynes, A. J., \& PAUleY, J. D. Heart rate and body temperature in separated monkey infants. Biological Psychiatry, 1978, 13, 91-105.

RICHARDS, J. E. The statistical analysis of heart rate: A review emphasizing infancy data. Psychophysiology, 1980, 17, 153-166.

Richter, C. P. On the phenomenon of sudden death in animals and man. Psychosomatic Medicine, 1957, 19, 191-198.

Rosellini, R. A., Binik, Y. M., \& Seligman, M. E. P. Sudden death in the laboratory rat. Psychosomatic Medicine, 1976, 38, 55-58.

Rosenmann, M., \& Morrison, P. Physiological characteristics of the alarm reaction in the deer mouse Peromyscus maniculatus bairdii. Physiological Zoology, 1974, 47, 230-241.

Ryden, 0. O. Heart rate response in great tit nestling Parus major to an alarm call. Journal of Comparative and Physiological Psychology, 1980, 94, 426-435.

Sampson, L. D., Schneiderman, N., Wallach, J., Gavin, W. J., \& Francis, J. S. Differential cardiovascular changes as a function of stimulation electrode site in rabbit hypothalamus. Physiology \& Behavior, 1977, 19, 111-120.
Schneiderman, N., Francis, J., Sampson, L. D., \& Schwaber, J. S. CNS integration of learned cardiovascular behaviour. In L. V. Dicara (Ed.), Limbic and autonomic nervous system research. New York: Plenum Press, 1974.

Seiler, C., Cullen, J. S., Zimmerman, J., \& Reite, M. Cardiac arrhythmias in infant pigtail monkeys following maternal separation. Psychophysiology, 1979, 16, 130-135.

UgLOw, R. F. Some effects of acute oxygen changes on heart and scaphognthite activity in some portunid crabs. Netherlands Journal of Sea Research, 1973, 7, 447-454.

Watson, W. H., \& WySE, G. A. Coordination of the heart and gill rhythms in Limulus. Journal of Comparative Physiology, 1978, 124, 267-275.

Wells, M. J. The heart beat of Octopus vulgaris. Journal of Experimental Biology, 1979, 78, 87-104.

WeLLS, M. J. Nervous control of the heart beat in Octopus. Journal of Experimental Biology, 1980, 85, 111-128.

Wiersma, C. A. G. Neurons of arthropods. Cold Spring Harbor Symposium on Quantitative Biology, 1952, 17, 155-163.

Wiersma, C. A. G., \& Novitski, E. The mechanism of the nervous regulation of the crayfish heart. Journal of Experimental Biology, 1942, 19, 255-265.

Wilkens, J. L., Wilkens, L. A., \& McMahon, B. R. Central control of cardiac and scaphognathite pacemakers in the crab Cancer magister. Journal of Comparative Physiology, 1974, 90, 89-104.

(Manuscript received March 4, 1981; revision accepted for publication July 16,1981 .) 